\title{
An Unexpected Meningitis Caused by Wooden Foreign Bodies in the Middle Ear: A Case Report

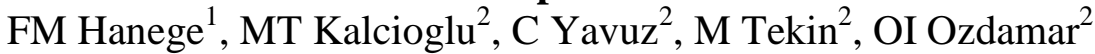

\begin{abstract}
Foreign bodies in the ear are often seen in infantile age group and are located on the external auditory canal. Foreign bodies of the middle ear however are quite rare. In this report we presented a 49-year-old white male patient who pushed dozens of wooden pieces to the middle ear himself and this situation was complicated by meningitis. We perfomed open cavity tympanomastoidectomy and removed the wood pieces from middle ear. Meningitis symptoms improved rapidly after surgery. Foreign bodies in the tympanum may result in dangerous complications such as meningitis. In cases of detected foreign bodies early intervention is extremely important to prevent this kind of fetal complications.
\end{abstract}

Keywords: foreign body, middle ear, meningitis, complication 


\section{INTRODUCTION}

Foreign bodies are common problems among ENT diseases $(1,2)$. In terms of locations of foreign bodies, ears are the first place with $44.3 \%$, followed by nose with $24.9 \%$, pharynx with $23.2 \%$, esophagus with $5.3 \%$ (3). Foreign bodies of ears are seen most often in children between 2-8 ages, and they are usually seen in external auditory canal (4). Foreign bodies are rare in the middle ear and cause some complications, if they stay in the middle ear for a long time.

\section{CASE REPORT}

A 49-year-old white male patient, diagnosed of meningitis by Infectious Diseases Clinic and admided to our clinic with complaints of ear discharge, dizziness, hearing loss. Medical history of the patient revealed two chronic otitis media surgical operations, one of them 13 years ago on the right ear and the other one 8 years ago on the left ear. Despite the ongoing topical medication for 10 days, he applied due to complaints of unresolved left ear discharge, dizziness and hearing loss. In his ear examination the left tympanic membrane could not be evaluated due to an oedematous left external ear filled with polyploidy soft tissue, and in its temporal bone thin cross-section computed tomography soft tissue density filling mastoidectomy defect and surrounding ossicles on the left ear and bone defect on tegmen tympani were detected (figure 1). Abscess was drained due to pre-diagnosed abscess, a minimal abscess material suppurated.

Revision canal wall-down tympanoplasty operation was performed under general anesthesia on the patient with the pre-diagnosis of ear rooted meningitis accompanied by the history of a previous open cavity tympanomastoidectomy. During the operation, dozens of wood pieces were detected in the middle ear (figure 2, 3). These pieces were placed around the 
ossicular into the middle ear and removed carefully. Meningitis symptoms improved rapidly after the surgery. As the overall situation improved, the patient was discharged. At the fourteen month follow-up of the patient no problems were observed.

\section{DISCUSSION}

Foreign bodies are commonly found in the external ear canal and beads, cotton tips, insects and paper are most common identified foreign bodies $(5,6)$. However, they are quite rare in the middle ear. Most common identified foreign bodies are Kohan at al reported 6 patients with detected hearing device matrixes settled on the middle ear (7). Foreign body in the middle ear causes othalgia, fullness, hearing loss, tinnitus and ear discharge complaints in patients. In ear examinations generally debris, granulation tissue, tympanic membrane perforation are detected on the external auditory canal $(8,9)$. In some cases foreign bodies can be detected on the perforated membrane (9). Imaging methods are useful in some cases to get information about the structure and localization of foreign objects (7-9). In our case, in contrast to frequently seen cases, the foreign body was placed in the middle ear, and caused ear discharge and dizziness complaints. In previous publications, it is reported that as in our case, the foreign body can be removed piece by piece, and in some cases require extensive surgery requiring removal of tympanic membrane and also ossicles $(7,10)$. Foreign body's long-time existence in the tympanum causes the development of complications. Chronic otitis media, mastoiditis, foreign body reaction coalescence mastoiditis and cholesteatoma are some of these complications (11). These complications may be accompanied by autogenous origin, such as meningitis, as we mentioned in our case. With the appropriate surgical initiative, foreign bodies should be cleaned 
out and source of infection should be eliminated. Likewise, in the presented case, patients implemented the canal wall-down tympanoplasty surgery, foreign bodies were cleaned out and the source of infection was eliminated.

As a conclusion, foreign bodies in the tympanum may result in dangerous complications such as meningitis. In cases of detected foreign bodies early intervention is extremely important to prevent these kind of complications. 


\section{REFERENCES}

1. Srinivas Moorthy PN, Srivalli $\quad$ M, Rau GV, Prasanth $\quad$ C.

Study on clinical presentation of ear and nose foreign bodies. Indian J Otolaryngol Head Neck Surg. 2012; 64(1):31-35.

2. Chavoshzadeh Z, Golnabi A, Rezaei N, Mehdizadeh M. Lareyngeal foreign body aspiration misdiagnosed as asthma: two case reports and a review of the literature. B-ENT. 2011; 7(2):137-140.

3. Chiun KC, Tang IP, Tan TY, Jong DE: Review of ear, nose and throat foreign bodies in Sarawak General Hospital. A five year experience. Med J Malaysia. 2012; 67(1):17-20.

4. Baker MD. Foreign bodies of the ears and nose in childhood. Pediatr Emerg Care. 1987; 3(2):67-70.

5. Ryan C, Ghosh A, Wilson-Boyd B, Smit D, O’Leary S. Presentation and management of aural foreign bodies in two Australian emergency departments. Emerg Med Australas. 2006; 18(4):372-378.

6. Dubois M, Francois M, Hamrioui R. Foreign bodies in the ear; report of 40 cases. Arch Pediatr. 1998; 5(9):970-973.

7. Kohan D, Sorin A, Marra S, Gottlieb M, Hoffman R. Surgical managment of complications after hearing aid fitting. Laryngoscope 2004; 114:317-322.

8. Eleftheriadou A, Chalastras T, Kyrmizakis D, Sfetsos S, Dagalakis K, Kandiloros D. Metallic foreign body in middle ear: an unusual cause of hearing loss. Head Face Med. 2007; 3:23.

9. Simons JP, Eibling DE. Tympanic membrane perforation and retained metal slag after a welding injury. Otolaryngol Head Neck Surg. 2005; 133(4):635-636. 
10. Jacob A, Morris TJ, Welling DB. Leaving a lasting impression: ear mold impressions as middle ear foreign bodies. Ann Otol Rhinol Laryngol. 2006; 115(12):912-916.

11. Panda NK, Verma RK, Jain A. Autotymapanomastoidectomy in a case of cholesteatoma with foreign body. Indian J Otolaryngol Head Neck Surg. 2011; 63(Suppl 1):68-70. 


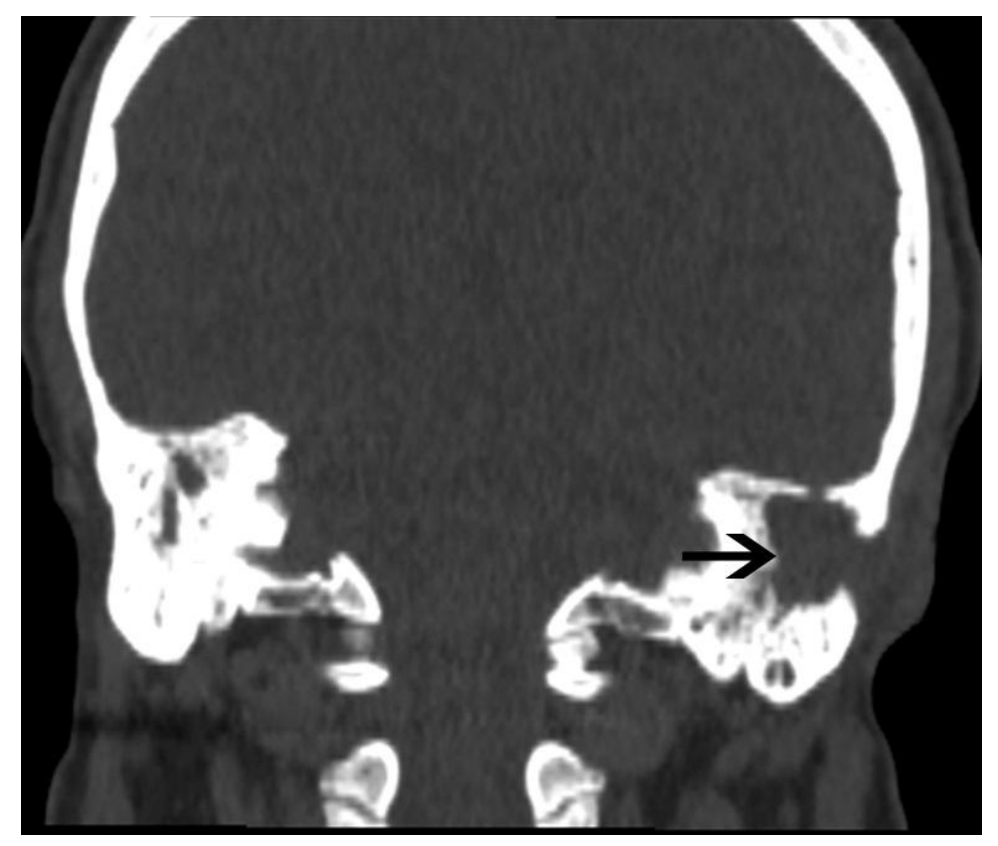

Figure 1: Mastoidectomy defect fills with soft tissue and bone defect on tegmen tympani

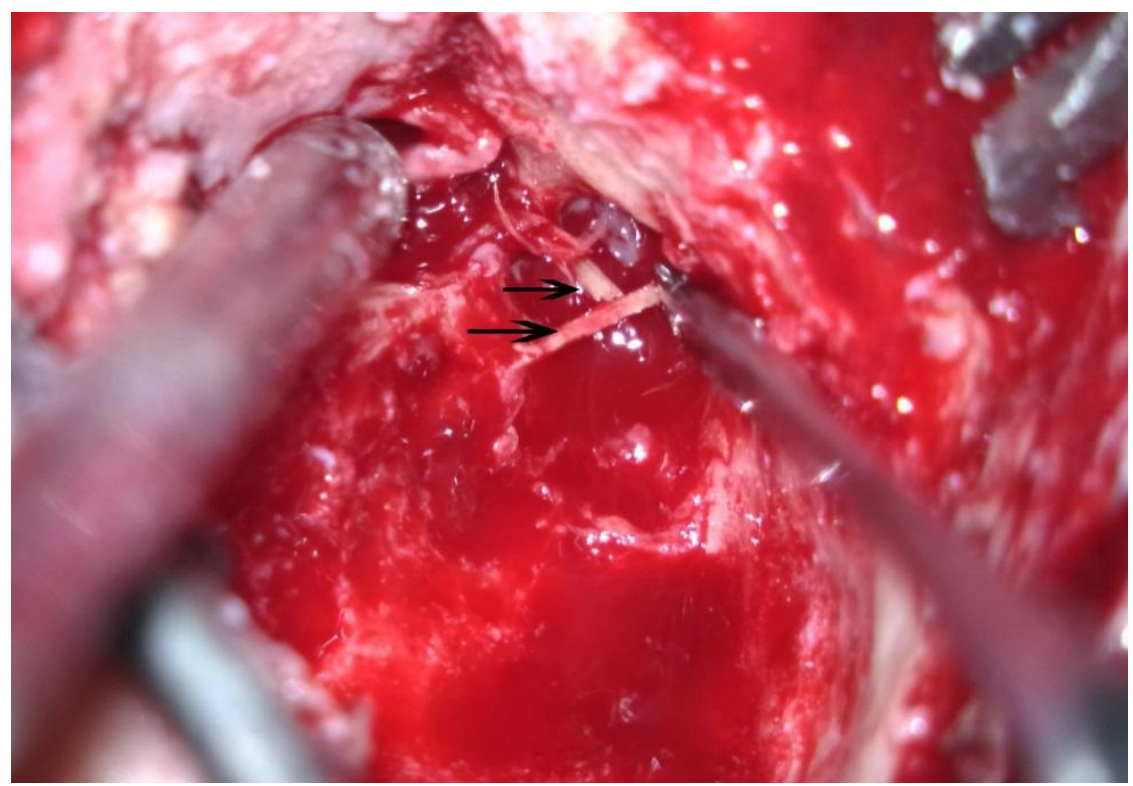

Figure 2: Pieces of wooden into the middle ear 


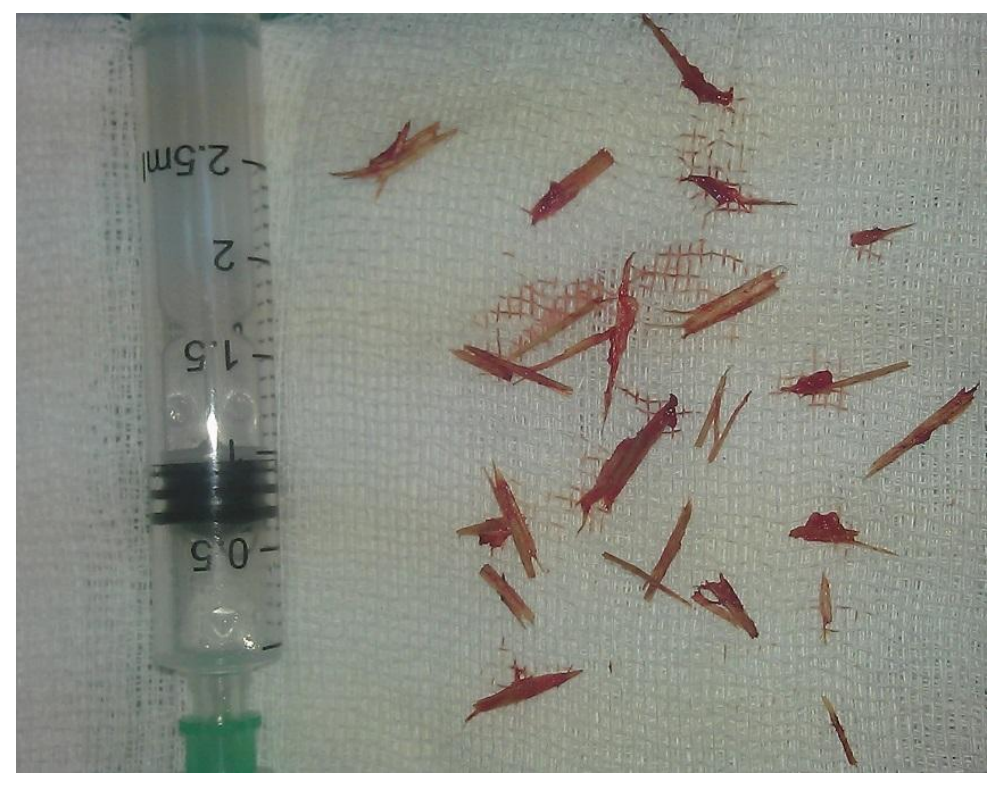

Figure 3: Pieces of wooden removed from the middle ear 